\title{
Wearables and Social Signal Processing for Smarter Public Presentations
}

\author{
ALAEDDINE MIHOUB, Orange Labs, Meylan, France \\ GREGOIRE LEFEBVRE, Orange Labs, Meylan, France
}

\begin{abstract}
Social Signal Processing ${ }^{1}$ techniques have given the opportunity to analyze in-depth human behavior in social face-to-face interactions. With recent advancements, it is henceforth possible to use these techniques to augment social interactions, especially human behavior in oral presentations. The goal of this study is to train a computational model able to provide a relevant feedback to a public speaker concerning his coverbal communication. Hence, the role of this model is to augment the social intelligence of the orator and then the relevance of his/her presentation. To this end, we present an original interaction setting in which the speaker is equipped with only wearable devices. Several coverbal modalities have been extracted and automatically annotated namely speech volume, intonation, speech rate, eye gaze, hand gestures and body movements. In this paper, which is an extension of our previous article published in IUI'17, we compare our Dynamic Bayesian Network design to classical J48/MLP/SVM classifiers; propose a subjective evaluation of presenter skills with a discussion in regards to our automatic evaluation; and we add a complementary study about using DBScan versus Kmeans algorithm in the design process of our Dynamic Bayesian Network.
\end{abstract}

CCS Concepts: • Human-centered computing $\rightarrow$ Human computer interaction (HCI) $\rightarrow$ HCI theory, concepts and models

\section{KEYWORDS}

Oral presentation; Social intelligence; Wearable devices; Multimodal behavior assessment; Dynamic Bayesian Network.

ACM Reference format:

Alaeddine Mihoub and Grégoire Lefebvre. 2018. Wearables and Social Signal Processing for Smarter Public Presentations. ACM Transactions on Interactive Intelligent Systems xx, xx, Article xx (xx 2018), 23 pages.

\section{INTRODUCTION}

Oral communication is considered one of the most valued interpersonal skills in variety of domains [37], such as education, business and politics [30]. This task of public speaking stresses many people and was featured in many surveys as their number one fear, even higher than death [50]. Fortunately, as stated in the literature of clinical psychology [11], this skill is not only a bestowed gift to minority of charismatic individuals but rather a skill that can be learned and improved. Nevertheless, being an efficient communicator requires a lot of practice and training notably with experts and professional coaches. It requires to continually modulate both verbal and coverbal cues, and to perceive, interpret and react to audience displays and signals. The nonverbal communication in particular is acknowledged to play a significant role in social interactions [3,28]. According to $[2,16]$ at least 65 percent of the information in conversations, are conveyed via nonverbal behaviors.

Permission to make digital or hard copies of all or part of this work for personal or classroom use is granted without fee provided that copies are not made or distributed for profit or commercial advantage and that copies bear this notice and the full citation on the first page. Copyrights for components of this work owned by others than the author(s) must be honored. Abstracting with credit is permitted. To copy otherwise, or republish, to post on servers or to redistribute to lists, requires prior specific permission and/or a fee. Request permissions from Permissions@acm.org.

2160-6455/2018/MonthOfPublication - ArticleNumber $\$ 15.00$

Copyright is held by the owner/author(s). Publication rights licensed to ACM.

http://dx.doi.org/10.1145/3234507 
Moreover, studies on conversational behavior have confirmed that these coverbal cues - such as body posture, hand gestures, facial expressions, eye gaze, speech energy, intonation and speech rate - are strongly involved in maintaining social attention and social glue [31]. Thus, mastering coverbal behavior in public speech is a key success for being a brilliant communicator that is what we call the social intelligence [1].

Social intelligence, as explained by Vinciarelli et al. [49], is one facet of our cognitive capabilities that guide us to interact harmoniously with others in different situations and contexts. In this paper we present a specific social face-to-face interaction carefully designed to model the social intelligence of a speaker in an oral presentation scenario. We present an original setting using only wearable devices in order to study the subtleties of human behavior in public speech. Our main challenge is to train a behavioral assessment model able to interpret the presenter nonverbal performances, estimate the cognitive state of the presenter and then predict an appropriate feedback. The feedback is aimed to enhance the awareness about coverbal attitude and thus the social intelligence of the speaker. In this work, the feedback modeling is solved in a data-driven way through a Dynamic Bayesian Network whose graphical structure describes the complex relationship between the cognitive state of the presenter, his multimodal performance scores and the adequate feedback. The longterm goal of our research is to implement our behavioral evaluation model on wearable devices and test in real-time the relevance of our modeling and the social acceptability of the setting. More generally, this research can be enlarged to many other domains besides public speaking such as job interviews, customer services and sales, communication for health-care professionals, helping people with social difficulties, etc.

Note that this article is an extension to our previous article published in [37]. In this paper, we complete that study with 3 main contributions, which are: a comparative study between our DBN design and classical J48/MLP/SVM classifiers; a subjective evaluation of presenter skills with a discussion in regards to our automatic evaluation; and a complementary study about using DBScan versus K-means algorithm in the DBN design process. The paper is organized as follows: the next section reviews the state of the art of feedback systems and models. In Section 3, we introduce our feedback generation model. In Section 4, experiments are conducted to show the relevance of our approach, both from an intelligent system perspective and from a user-centric study. Results are exposed and discussed in Section 5 and finally Section 6 summarizes our contributions.

\section{RELATED WORKS}

This research is part of the automatic analysis of human behavior in social interactions [13]. Automatic conversation analysis tries to infer from raw signal information about social actions, social emotions, social relations and social attitudes [49]. Depending on the application domain, several computational models have been proposed to cope with these issues. For instance, Mihoub et al. [35] presented a behavioral model designed to endow social robots with social skills enabling them to engage relevant interactions with human interlocutors. The model was based on Hidden Markov Models and was able to analyze human-partner behavior and generate adequate coverbal behavior particularly eye gaze fixations. Nihei et al. [41] established classification models that estimate influential statements in group discussions using prosodic features, head motion and eye gaze information. Group discussions are used in some countries for job recruitment processes. Expert recruiters observe the social behavior of all candidates and select only the good discussion performers. Through their modeling, Nihei et al. aimed to elaborate automatic skill evaluation through automatic scene analysis. They showed that persons who make influential statements frequently present better facilitation ability in discussions. To enhance group collaboration, Kim et al. [27] introduced the Meeting Mediator (MM), a real-time system that detects social interactions in meetings and provide feedback on the interaction dynamics. The exchanged social signals - based essentially on speech features and body movements - were captured by Sociometric portable badges and visualized on mobile phones of the interacting interlocutors. They showed that the system was able - in a brainstorming scenario - to ameliorate interactivity level and to reduce behavioral differences between non-dominant and dominant people. 
Besides promoting social dynamics in-group meetings, one major application of social augmenting is to enhance social behavior in public speak. In this context, three configurations are identified in the literature: (1) Behavior assessment in training sessions, i.e. the feedback is provided before a real presentation situation. (2) Feedback provided online, i.e. during a real presentation situation. (3) Feedback provided offline, i.e. after a real presentation situation.

The presentation trainer [43] is a recent example of systems within the first configuration. It is a multimodal tool designed to track the user's body and voice (body posture, gestures, volume, pauses, etc.) using a microphone and a Kinect ${ }^{\circledR}$. The feedback is transmitted through a visual graphical interface and a connected wristband (haptic information). Two types of feedback (corrective and interruptive) were provided using a rule-based model. For the latter type, the system considers the user's mistake as severe and interrupts the speaking. To reduce the cognitive load of the presenter only one feedback is instructed at a time and the system was demonstrated to have significant effect on users' learning. $\mathrm{MACH}$ (My Automated Conversation coacH) $[21,22]$ is another well-known system in the literature. Through a virtual agent the system simulates a job interview with human participants. At end of a session, a summary is provided concerning many aspects of the nonverbal communication such as total pause duration, speaking rate, weak language (filler words such as "basically" and "umm"), pitch variation, smiles, head gestures, loudness, etc. In the same context, Chollet et al. [5] explored an interactive virtual audience paradigm to create a forgiving and engaging environment for public speak training. The virtual audience was projected on a life-size screen and was animated by a wizard of oz strategy. The system was able to give direct visual feedback on the screen but also implicit feedback via the nonverbal behavior of the animated characters. For instance, a character could exhibit a forward or a backward posture depending on speaker's performances. The system was shown to have positive effect on learners' audiovisual behavior.

The second type of systems proposed in the literature, are those delivering online feedback during real presentations. Knowing that human attention is a limited source [44] and that human brain is not adept for multitasking [41, 45], the challenge is to convey a relevant feedback in a convenient format and with an optimal frequency, so that speakers are not distracted from their task. For instance, Rhema [47] is an intelligent user interface for wearable Google ${ }^{\circledR}$ glasses, developed to help presenters modulate speech volume and speech rate during their talk. The paper shows that optimal feedback should be one instruction at a time and in single words format (e.g. louder, faster, etc.). One limitation of this work is the lack of multimodality in the behavior assessment. Logue [7] is another system designed to deliver in-situ real-time feedbacks about the nonverbal behavior of a speaker, especially about speech rate, body energy and openness. The social sensing is performed by a microphone, a Kinect ${ }^{\circledR}$ placed in front of the speaker and wearable Vuzix ${ }^{\circledR}$ glasses which were used also for feedback rendering. The feedback generation was provided by a rule-based model and a set of manually fixed thresholds. With two user studies, the authors showed that Logue system was perceived to be helpful, and that it has got significant impact on users' performances.

The multi-sensor self-quantification framework [12] is a feedback system belonging to the third aforementioned configuration. It generates an offline report about many aspects of the oral presentation such as speaking rate, liveliness, body movement, gestures, speaker attention, audience engagement, etc. It utilizes a whole motion capture system composed of two static cameras, one Kinect $\AA^{8}$, and three wearable Google ${ }^{\circledR}$ glasses (one for the user and two for public). The feedback model was based on Support Vector Machine models trained with annotated multimodal data. The ground truth was collected using manual annotation of videos segmented in multiple 10-second clips. The user study showed that the analytic report generated after each presentation received positive opinions from the experiment participants.

Compared to literature, our research paradigm is to offer the final user the advantages of the three configurations. In fact, in our study we claim the following contributions: 
- An original social sensing setting, not based on classic motion capture systems but only on wearable devices that are easily portable and susceptible to be shortly available for general public.

- At this state, our system offers training and practice possibilities as well as detailed offline reports on behavioral performances. It is intended to integrate real-time feedbacks in the near future.

- Behavior evaluation is performed to be as exhaustive as possible in order to deal with almost coverbal modalities (speech features, hand/body motions and eye gaze).

- Contrary to existing feedback models (rule-based, classic classifier, wizard of oz, etc.), we propose an evaluation model based on a probabilistic graphical model (DBN) that is more appropriate - thanks to its internal characteristics - for modeling temporal complexity of human behavior dynamics.

\section{FEEDBACK MODELING}

As introduced, we propose to solve the feedback modeling in a data-driven way through a Dynamic Bayesian Network whose graphical structure describes the complex relationship between the cognitive state of the presenter, his multimodal performance scores and the adequate feedback. We remind that the main challenge of our model is to coach social intelligence to public speakers. Before exposing our DBN modeling for behavior assessment and feedback generation, we start in the next paragraph with a brief review on DBNs.

\subsection{Dynamic Bayesian Networks}

Bayesian Networks (BNs) [40] belong to the family of Probabilistic Graphical Models (PGMs) [29]. PGMs have married in a same formalism graph theory and probability theory in order to provide intuitive and effective tools to represent a joint probability distribution over a set of random variables [26]. Formally, a Bayesian Network (BN) is a directed acyclic graph whose nodes are random variables and whose edges represent conditional dependencies between these variables. An edge linking a parent node $\mathrm{X}$ to a child node $\mathrm{Y}$ semantically means that node X exerts direct (or causal) influence over node Y. Dynamic Bayesian Networks (DBNs) [39] have extended static Bayesian Networks (BNs) by modeling the temporal evolution of the variables. Beyond intuitive graphical representation and uncertainty dealing, they efficiently model complex temporal relationships between variables. Since we consider processes that are only Markovian (i.e. variables at slice $t$ are only dependent on $t-1$ slice variables) and stationary (i.e. conditional probability distributions do not change over time), a DBN can be sufficiently depicted by a 2-timeslice structure called 2-timeslice Bayesian Network (2-TBN) (see an example in Fig. 1) [14]. Due to their multiple characteristics, DBNs have been used successfully in many domains; in particular, they represent an attractive and a powerful formalism for modeling dynamics of human behavior in social interactions $[23,36]$.

\subsection{DBN-based models}

3.2.1 Cognitive state. In this work, we propose to estimate the cognitive state (CS) of the presenter to our modeling in order to get better prediction of feedbacks. In fact, additional to annotated variables described in Section 4, we introduced a novel variable (CS) that tries to model the cognitive state of the speaker. Cognitive states condition perception-action loops of the human behavior: they reflect cognitive processes that coordinate modalities of human behavior but also contextualize the interaction by considering its circumstances and its evolution. Examples of CS could be "informing", "nodding", "listening", "turn taking", etc. Notice that one single CS could also sequence many sensory-motor states together. Similar concepts have been proposed in the literature of multimodal behavior modeling [23, 32, 35]. In our application, we used data mining techniques to explore the cognitive states of the presenter because an exhaustive state list cannot be determined a priori. Actually, we used many clustering algorithms to infer these states from both multimodal scores and feedbacks. The multimodal scores here represent the evaluations of the different modalities of the speaker's behavior. In other words, each score is an assessment of the speaker's performance according to one modality (such as volume, intonation, speech rate, gaze, gesture energy and body energy). Indeed, associating scores and feedbacks this way, allows the estimated states to better contextualize interaction phases. 
3.2.2 Proposed Model. Based on Dynamic Bayesian Networks (DBNs), our model uses machine learning techniques to intrinsically associate CS and input multimodal performance scores to the final annotated feedbacks. The main objective is to retrieve annotated feedbacks and to prove usefulness of this model for future online coaching system. List of used variables, as well as their respective cardinalities were as follows (see more details in Sections 4.5 and 4.6):

- CS: cognitive state of the presenter, 27 states,

- V: Volume, 3 levels (low, good, high),

- I: Intonation, 3 classes (monotone, medium, lively),

- S: Speech rate, 3 levels (low, good, high),

- G: Gaze fixations, 3 classes (improvable, medium, good),

- H: Hand gesture energy, 3 levels (low, good, excessive),

- B: Body energy, 3 levels (low, good, excessive),

- F: Feedback, 9 types (volume+, liveliness+, speech_rate+, speech_rate-, public+ (gaze), gesture+, body+, none, good).

Our proposed model is illustrated in Fig. 1. The internal structure of our DBN was designed to efficiently model causal relationships between different nodes. This causality graph presents interesting properties:

- The cognitive state of the speaker influences his multimodal behavior (blue arrows).

- The appropriate feedback is a direct consequence of multimodal scores of nonverbal behavior (green arrows).

- The appropriate feedback is also directly influenced by the mental state of the speaker (red arrows).

- The network reflects the temporal correlation between cognitive states since a CS at a certain time is influenced by the previous state of the speaker (grey arrows).

- The network reproduces an important instruction that was given to annotators concerning feedback; this instruction has incited annotators to diversify feedbacks by considering feedback history (cf. Section 4.6). Looking to the structure, we can easily identify temporal dependence between feedbacks (black arrows).

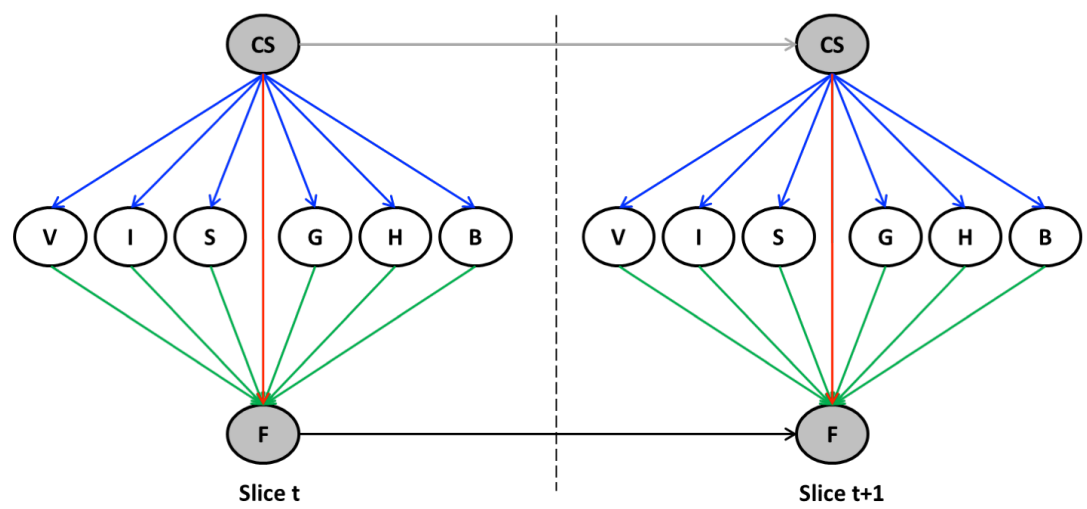

Fig. 1. Our proposed DBN model (2-TBN model). Variables with white background are observable while grey ones represent variables to predict.

To demonstrate the efficiency of the proposed causality network, we compare in Section 5 our principal model with a second baseline DBN whose structure does not consider the CS of the presenter (cf. Fig. 2). 


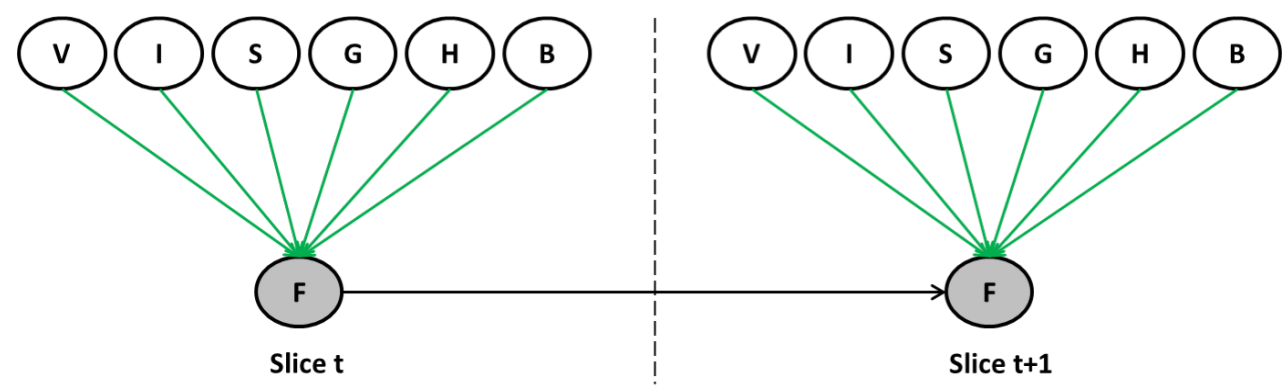

Fig. 2. Baseline model: DBN structure without cognitive state (CS) modeling.

\section{EXPERIMENT DESIGN}

\subsection{Scenario}

The objective behind our proposed experiment is to collect multimodal behaviors of many speakers in an oral presentation scenario (cf. Fig. 4). The presenters were asked to give a "pitch" presentation for 5 minutes. Each presentation was composed of 5 slides in which each slide corresponds to one of the following items: presenter education, professional experiences, technical/interpersonal skills, current projects, and future projects. In our experiment, this pitch scenario was particularly chosen since it highly motivates participants to be at the top of their performances compared to other presentation topics.

\subsection{Protocol}

11 presenters ( 7 male and 4 female) were recruited to participate in our experiment. Our study was limited to French native speakers in order to have homogeneous behavioral patterns and a standard annotation protocol. Participants' ages ranged from 22 to 48 years old with an average of 34 . They had different functions: interns, developers, PhD students, researchers, ergonomists and project managers (cf. Fig. 3). Two weeks before recording, participants were asked to fill an online form about (1) their demographic information, (2) selfassessment of skills in oral presentations and (3) their personal Curriculum Vitae. On a 10-point scale question ( $1=$ never, $10=$ very frequent), our participants rated themselves on average 5.36 for frequency of giving presentations, $6.27(1=$ not at all, $10=$ very comfortable $)$ for their comfort in public speak and $6.45(1=$ very bad, $10=$ very good) on how good they think their nonverbal communication is. Experimenters used the third information (i.e. personal CV) to build presentation slides that were sent to participants one week before their talk. This protocol helped us to have standard presentations for all speakers, which neutralizes preparation effects on behavior performances. Each presenter was asked to give two presentations on two different days to minimize learning effect, which leads to a total of 22 presentations. These presentations were recorded in 7 sessions with groups of 3 or 4 participants. Although the moderate number of participants, we think that the data set is sufficiently rich and complex thanks to the multimodality of captured signals and the strictness of the protocol. This dataset completely fulfills the objective of capturing the microstructures and regularities of speaker behaviors and attitudes. The mean length of a session was about 1 hour and 15 minutes. The groups were designed to mix the 11 participants in different groups. Since we have two presentations per participant, this protocol avoided presenters' lassitude caused by the repetition of a talk in front of the same person. For each group (for instance a 4-person group), we have one presenter and three observers who constitute the audience.

At the beginning of each session, the experimenter presents the study and its progress phases. He also takes few minutes to expose the importance of nonverbal communication in a public speak and gives some recommendations about it. This step guarantees that participants have similar baseline knowledge about coverbality in public speak. 

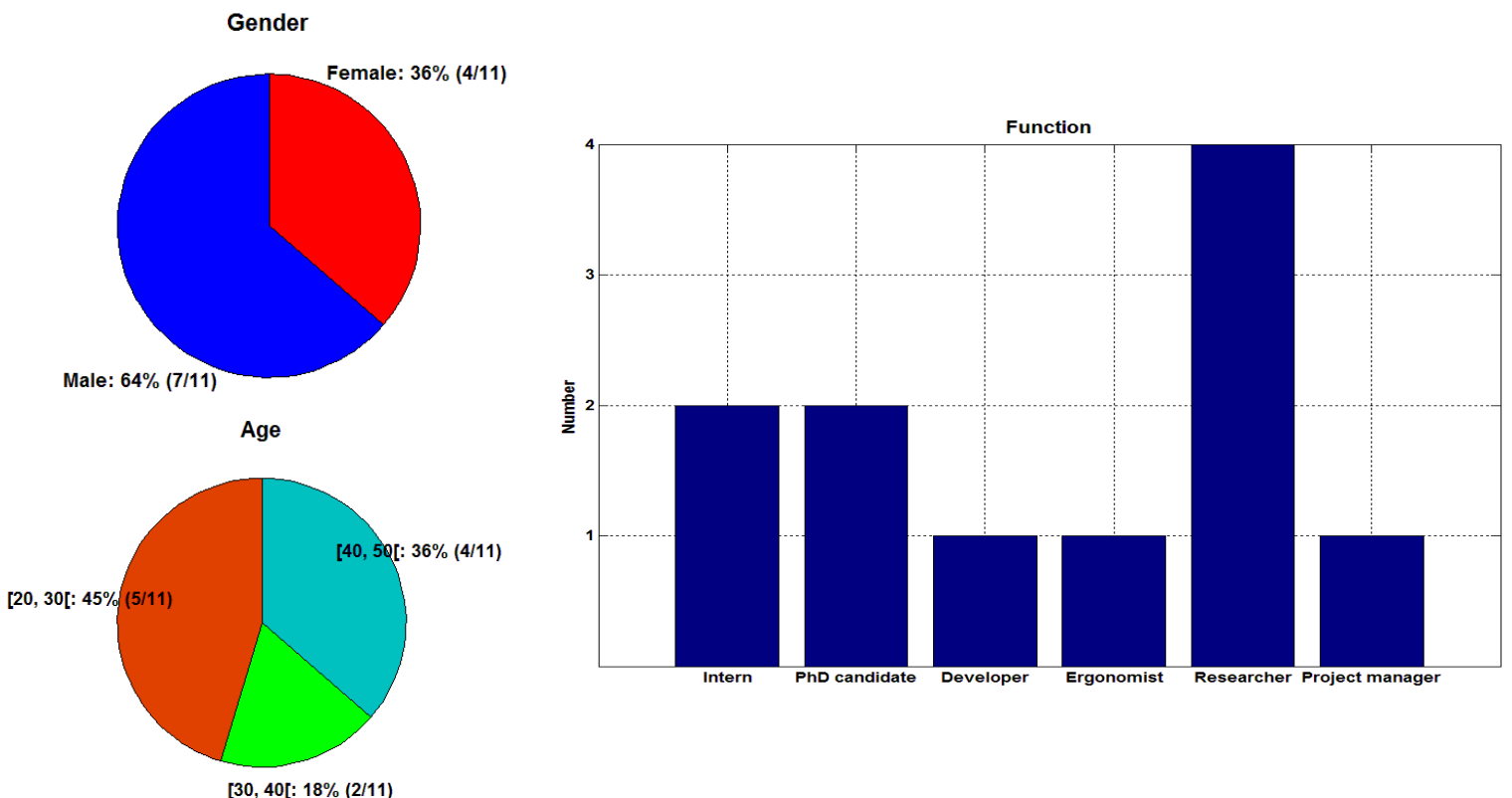

Fig. 3. Experimenter repartitions by gender, age and function.

After each presentation, one form is filled by the speaker for self-assessment and a second form is filled by an audience member that we call the evaluator. Two tablets were used to this end; collected information from these questionnaires will be discussed in the post-study experiment section. After each presentation, a role changing strategy is applied by the experimenter so that each person participates as a presenter but also as observer for the rest. Particularly, he/she will be an evaluator for a specific speaker by rating his/her behavior performances through the second questionnaire. Each evaluator was discreetly informed from the beginning about the person to evaluate. Note that, our changing role strategy ensured that two persons couldn't evaluate theirself mutually. The evaluators were instructed to pay attention to the coverbal behavior of the speakers especially voice, gaze, and body language.

\subsection{Experimental Setting}

The aim of the setting (cf. Fig. 4) is to sense the audiovisual social behavior of a presenter particularly voice attributes (volume, intonation, speech rate), eye gaze fixations, hand gestures and body movements. In order to make our evaluation system portable and usable in different contexts, only the presenter was equipped with wearable devices (cf. Fig. 5). For same reasons, we omitted the use of heavy motion capture systems by utilizing only wearables in part already democratized for public. Therefore, the multimodal behavior of a presenter was captured by:

- A smartphone (Samsung $\left.{ }^{\circledR}\right)$ put in the pocket of the presenter, used to track body movements.

- A smartwatch $\left(\right.$ Sony $\left.{ }^{\circledR}\right)$ worn on the directional hand, used to track hand gestures.

- Smart glasses (Epson Moverio BT-200®), a head-mounted device used to capture eye gaze fixations.

- A microphone connected to the glasses, used to monitor the presenter's speech signal.

For the purpose of annotation, we also equipped the scene with a static camera in order to film the whole interaction. 


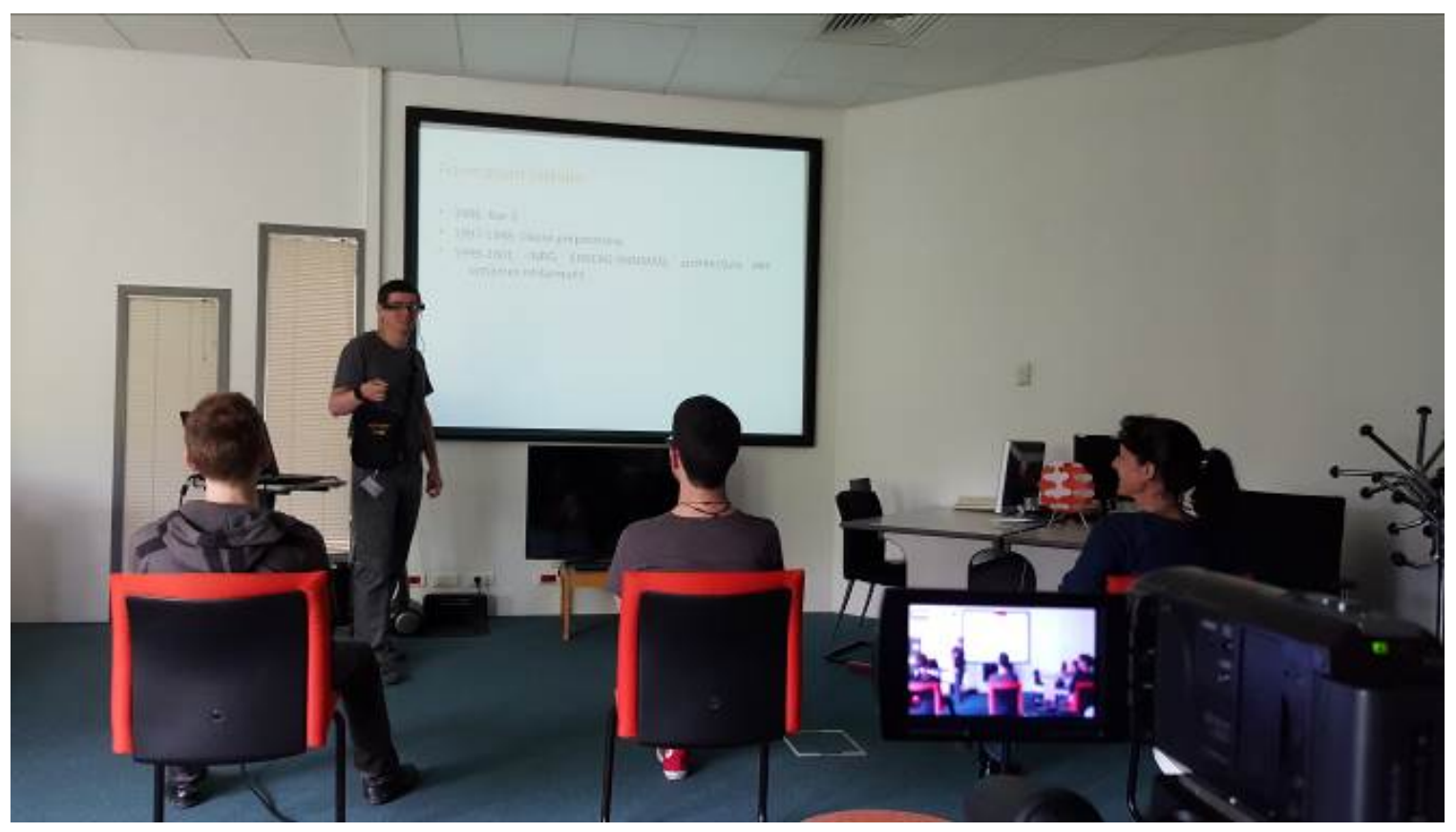

Fig. 4. Experimental setting of a presentation.

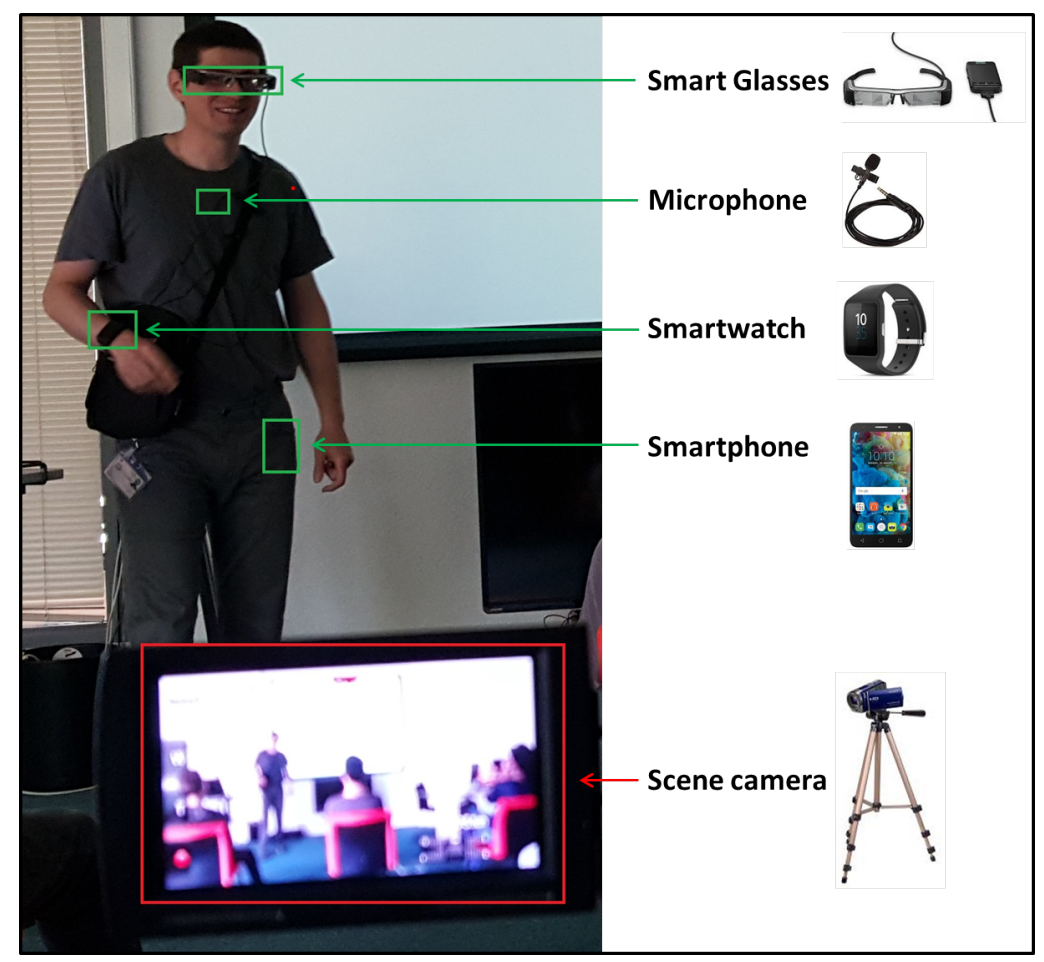

Fig. 5. List of devices used to capture presenter's behavior. 


\subsection{Recorded Signals}

For data acquisition, a recording platform was developed in order to collect multimodal and synchronized data from all devices. The recorded signals are as follow:

- Accelerometer and gyrometer data from the pocket smartphone,

- Accelerometer and gyrometer data from the smartwatch,

- Accelerometer and gyrometer data from the glasses,

- Egocentric video scene from the front camera of the glasses,

- Speech signal from the microphone,

- Video environment from the static camera.

Human behavior is paced by subtle temporal coordination between different modalities. Thus, the challenge for our recording platform was to collect those signals in highly synchronized way; otherwise all social behavior modeling will be invalid. To this end, two Android® ${ }^{\circledR}$ applications were developed to handle recordings. The first application runs on the experimenter smartphone (Samsung®). It allows triggering recording for both presenter smartphone and smartwatch. The second Android® application monitors the glasses and the connected microphone. The two applications were controlled by the experimenter to help orators focus on their presentation task. Note that manual claps are also performed before and after each presentation in order to synchronize precisely the different modalities especially audio and video.

\subsection{Input Data Annotation}

Similar to many previous works [12], we chose to segment each presentation on 10-second clips. This frame granularity was used for all modalities in our annotation process. The mean duration of the presentations was about 37 frames (i.e. 6 minutes and 10 seconds long) and the overall time of presentations was about 2 hours and 15 minutes. The experiment design was thought to handle a list of selected modalities known to largely contribute on public talk pertinence. Next paragraphs describe the set of signal processing based rules utilized to infer these modalities from recorded signals.

4.5.1 Volume. A principal characteristic of human voice is volume. Known also as intensity or speech energy, the volume represents the loudness of an audio signal and plays fundamental role for transmitting clear messages for audience [10]. To analyze volume, we used an application based on the intensity extraction method of the Praat software [4]. We extracted also silent intervals for all segmented clips with a silent threshold of $-30 \mathrm{~dB}$, minimum silent interval duration of $0.1 \mathrm{sec}$ and minimum sounding interval duration of 0.05 sec. For each 10 -second clip, we first delete silent intervals and we calculate the mean from remaining intensities. Then, using two intensity thresholds [17] (50 dB and $60 \mathrm{~dB})$, the volume of the interval is classified into three categories: low, good and high. Thus, volume levels under $50 \mathrm{~dB}$ were considered as low while volume levels above $60 \mathrm{~dB}$ were considered as high. Note that these thresholds may slightly change depending on room noise and distance from audience.

4.5.2 Intonation. One major attribute of human prosody is intonation. It represents the way speakers modulate their voices and plays significant role for retaining audience's attention [10]. Pitch is known to be the main acoustic feature that correlates with intonation. Hence, for intonation analysis, we automatically extract pitches using an application based on Praat software [4]. For the extraction, we used a minimum pitch of $75 \mathrm{~Hz}$ and defaults parameters of the Praat autocorrelation method. To quantify pitch variation, it is possible to use the raw pitch standard deviation as a metric. However, because of differences between speakers especially males and females, this metric will give invalid and unfair comparison results [20]. Therefore, as proposed by R. Hincks $[19,20]$ the standard deviation should be expressed as a percentage of the pitch mean. This normalization is performed by dividing the pitch standard deviation by the mean; the resulting quotient is known by the acronym PVQ (Pitch Variation Quotient). Note that in Hincks works, the 
PVQ is also calculated for 10-second clips. In our work, we first calculate for each 10-second segment the corresponding PVQ. Then, using two thresholds inspired from [19] (0.10 and 0.23), the intonation is classified into three categories: monotone, medium and lively. In this way, PVQ values under 0.10 characterize monotonous speech while values above 0.23 reflect lively voice.

4.5.3 Speech rate. Balanced speech rate is another important attribute featuring voice quality. In fact, a slow rate may cause boredom while an accelerated rate may cause incomprehension and ambiguity. To measure speaking rate one possibility is to use the word per minute (WPM) metric. Nevertheless, because of major difference in word length, this measure can be imprecise in many contexts [15]. Expressing speech rate in syllables per second (SPS) solves this problem and presents many advantages. It allows a local analysis of voice and more efficient track of its rate variations [20]. In our work, the SPS measurement is used to characterize speech rate. In particular, we relied on Jong and Wempe algorithm [25] to segment our audio clips into syllables. The speech rate is then computed by dividing the number of detected syllables by the length of clips, i.e. 10 seconds. Afterwards, using two thresholds inspired from [8] (5.8 sps and 10 sps), the speech rate is classified into three categories: low, good and high. Accordingly, SPS values fewer than 5.8 feature low speaking rates while values above 10 distinguish highly speech rates.

4.5.4 Gaze. Besides retaining their attention, sustained eye contacts with listeners make the orator look engaged, more believable and more convincing. Further, it helps the presenter to better receive listeners' nonverbal signals (e.g. facial expressions) and then, responds and enhances his message deliver. In our work, eye gaze performances were annotated semi-automatically. First, using the videos of the glasses, we annotated manually fixations over four regions of interest: audience, personal computer, projection screen and elsewhere. From these annotations, we computed for each 10-second segment a gaze distribution that contains the percentage of each region of interest. Then we established a rule-based model that uses a list of thresholds in order to automatically classify the gaze segment into three categories: improvable, medium and good. At this state, fixations are annotated manually but we intend in the future to use computer vision techniques to automatically detect regions of interest. In particular, we may use accelerometer and gyrometer data of the glasses - coupled with visual features - in order to get better estimations. We think that is possible to have reliable estimation using this method, especially that our regions of interest are enough distinct (audience, personal computer, etc.) to be detected from head movements (extracted from the MEMs sensors of the glasses).

4.5.5 Gestures. Hand and body movements are known to be powerful tools to enhance social influence. Hand gestures for instance help to add emphasis and clarity to spoken words. Body movements help also to reinforce verbal messages and further ameliorate audience attraction [48]. In our setting, hand and body movements were sensed by wearables devices. In particular, hand gestures were inferred from accelerometer data from the smartwatch, while body movements were extracted from accelerometer data from the pocket smartphone. Firstly, for both modalities, a high-pass filter was applied to all axes to eliminate gravity force. In fact, a low-pass filter was used to isolate gravity force and then a high-pass filter is used to remove that gravity. Secondly, another low-pass filter (beta=0.8) is applied to reduce noises. Finally, using the 10 -second segments, we computed an energy measurement - based on effective power of signal [34] - in order to characterize gesture energy and body energy of the speaker. Based on two thresholds, the gesture energy was classified into three categories: low, good and excessive. Similarly, two other thresholds were used to assign body energy to one of those classes. Notice that in our annotation, we used only accelerometer data to describe overall energy of the presenter. In the future, we may also exploit gyrometer data in order to get more precisions on types and categories of gestures.

\subsection{Output Feedback Annotations}


After analyzing all videos, 9 specific feedbacks about nonverbal behavior of the presenter were proposed with an audience point of view:

1. "volume+" : louder speech volume,

2. "liveliness+" : more voice liveliness, used when intonation is perceived as monotone,

3. "speech_rate+" : faster speech rate,

4. "speech_rate-": lower speech rate,

5. "publict" : more eye contacts with the public,

6. "gesture+" : more hand gestures,

7. "body+" : more body movements,

8. "none" : no feedback, used when it is not appropriate to deliver a feedback at that moment,

9. "good" : used when nonverbal behavior is evaluated as good.

Notice here that the choice of each feedback - regarding the corresponding modality - is highly correlated to the context of oral presentation and the more frequent errors of public speakers. For instance, taking the volume modality as an example, the more frequent error in presentations context is 'very low volume'. Thus, for that modality, the selected feedback was volume+, i.e. louder speech volume. The same methodology is applied for the rest of modalities. As mentioned before, human brain is not adept for multitasking $[41,45]$ for this reason we chose to deliver only one feedback per 10-second segment. Efficiency of this paradigm has been already demonstrated in the literature [42, 46]. Each 10-second clip was affected to one of those feedbacks by two expert annotators that have strong background on co-verbal behavior research and particularly an excellent knowledge about nonverbal communication in public speech.

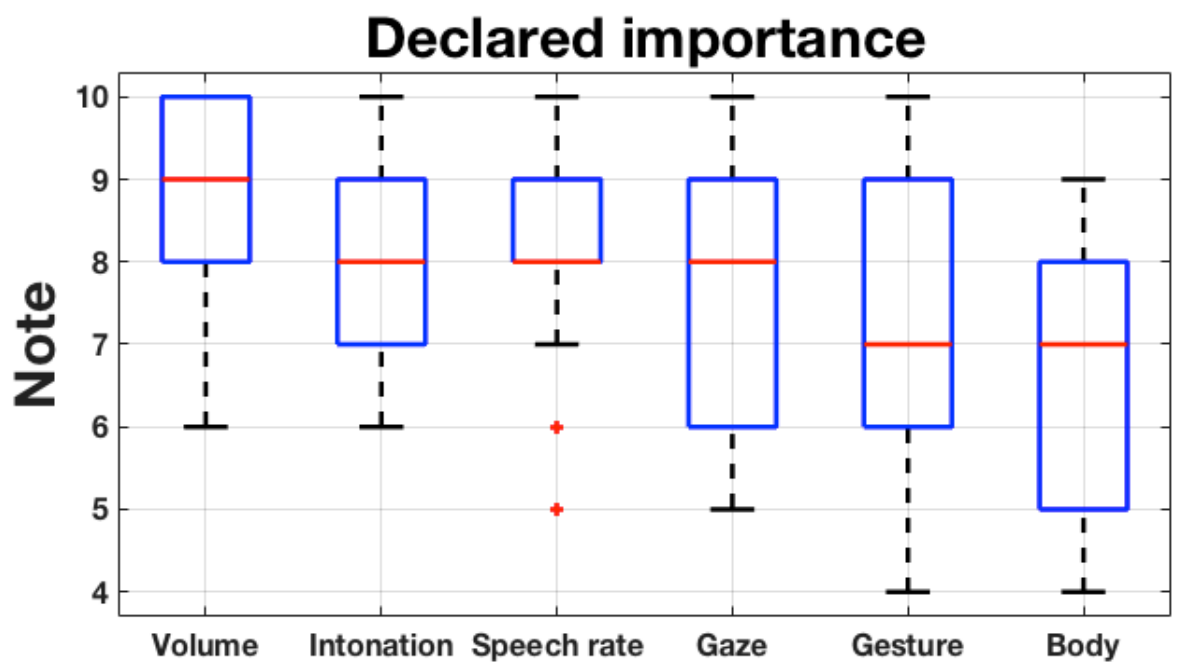

Fig. 6. Declared importance (from audience) of coverbal modalities for presentation relevance (1= not important at all, $10=$ very important). Voice is underlined the most important modality especially volume. Gaze fixations are also emphasized but with more diverging opinions. Finally, gestures and body movements are perceived as significant but less crucial.

ELAN software [45] was used to visualize and to annotate each presentation. Annotators were instructed to give priority firstly to voice attributes and gaze features, and secondly to hand gestures and body movements. These instructions were based on results from the post-experiment study. In fact, participants were asked 
about importance of each modality in public presentation and the final statistics (cf. Fig. 6) showed that voice features, as well as gaze, were declared more important than hand and body energies. It is also very important to encourage speakers with 'good' labeled feedbacks and stop feedback if everything is OK (i.e. using the 'none' label). Annotators were also instructed to consider the annotated feedback history during the annotation process in order to get diversified feedbacks and avoid sustained emphasis on a particular modality. Note that in case of mismatch between annotators, they were invited to debate on video segment and choose only one final feedback. We remind that the proposed DBN models - in the Feedback modeling section (cf. Section 3) - are mainly designed to retrieve these annotated feedbacks from automatic multimodal scores.

\section{EXPERIMENTAL RESULTS}

\subsection{Quantitative Results on Feedback Prediction}

Bayes Net toolbox [38] was used for DBNs learning and feedback estimation. EM algorithm [9] is applied for training while Junction tree algorithm [6] is applied for inference. The Expectation-Maximization (EM) algorithm is well adapted to our training problem since it represents an iterative method to estimate parameters of statistical models with latent variables, which is the case of DBNs. Junction tree algorithm is well adapted too to our model since we have only discrete observations and since it gives an exact solution for the inference problem (see [26] for more details about learning in graphical models). In particular, online inference is used for prediction, i.e. the feedback value $(F)$ at a slice $t$ is estimated only with the 6 observable data $(\mathrm{V}, \mathrm{I}, \mathrm{S}, \mathrm{G}, \mathrm{H}, \mathrm{B})$ till that instant t. Offline inference would rather use the total sequence, which is not appropriate for real-time systems. We remind that we have 22 sequences (11 participants and 2 presentations per participant). Using a leave-2-out-cross validation, we end up with 11 models; each model is trained on sessions of 10 participants and then tested on the two sessions of the remaining participant. Two metrics were used to quantify models performances. First, we used the precision of prediction, which corresponds to the percentage of retrieved feedbacks from original sequences. Because we are comparing similarity between two sequences, we use also a second approach with F-measure values based on Levenshtein distance [33]. In fact, Levenshtein distance computes a minimum number of operations to transform one sequence to another. From this optimal alignment the F-measure metric is directly computed. This method is more adequate to our problem since it tolerates small miss-alignments between original and predicted values. Indeed, speakers prefer to receive good feedbacks with light delays than incorrect feedbacks.

The following table values (cf. Table 1, Table 2, Table 3 and Table 4) correspond to calculated mean precision and F-measure values on the 11 trained models. Our first results are evaluated on different configurations. First, we chose two values for the temporal granularity of our multimodal data (i.e. 5 and 10 seconds per time slice). For instance, with a granularity of 10 seconds per time slice, mean duration of sequences filled to our models was 37 frames. Secondly, we chose two input data records: a standard vector with volume, intonation, speech rate, gaze, hand gestures, body movements and feedback features; and a compact vector with voice, gaze, movement and feedback features. As, we got 3 values per input modality and 9 possible feedback values, the input data dimension is $\{3,3,3,3,3,3,9\}$ for the standard input record. When we compact the acoustic information (volume/intonation/speech rate) and the movement features (hand/body movements), we obtain an input data dimension of $\{27,3,9,9\}$ for the compact input record.

5.1.1 Classifiers Results. For the sake of comparison with our DBN-based strategies, we implement others classifiers: Decision Trees (J48 algorithm), Multi-Layer Perceptron (MLP) and Support Vector Machine (SVM) with Weka software [18] (SMO algorithm for SVM). Table 1 presents the first results with a best F-measure of $65.93 \%$ using the standard features and a granularity of 10 seconds. The SVM classifier obtains these results. We can observe that for each tested classifier, the standard input record helps to get better results. For 
instance, the SVM classifier increases the F-measure values from $63.09 \%$ to $65.93 \%$ when using respectively the standard inputs and the compact inputs. Therefore, the compactness of information about acoustic and movement features seems to produce a lack of description impacting the final classification. The relative gain obtained by the SVM classifier may be justified by the size of the data to be learned which is quite small and the limited data dimension.

Table 1. Average performances for classifier-based feedback estimations (best results in bold format).

\begin{tabular}{lll}
\hline Granularity 10s & Precision (\%) & F-measure (\%) \\
\hline Decision Tree (Compact) & 58.51 & 64.32 \\
Decision Tree (Standard) & 60.37 & 65.30 \\
MLP (Compact) & 59.88 & 65.06 \\
MLP (Standard) & 60.49 & 65.19 \\
SVM (Compact) & 57.79 & 63.09 \\
SVM (Standard) & $\mathbf{6 1 . 8 5}$ & $\mathbf{6 5 . 9 3}$ \\
\hline
\end{tabular}

5.1.2 DBN Results. We develop different strategies to evaluate DBN-based training. Two strategies are presented in the following: firstly, a simple one without cognitive state (cf. Fig. 2) and secondly, a latent one with cognitive state modeling (cf. Fig. 1).

Table 2 presents the first DBN-based feedback estimations. The best performance is done with a latent strategy on standard input data and a granularity of 10 seconds per time slice. The corresponding average Fmeasure is $65.80 \%$ and the mean precision value is $60 \%$. It appears then that adding a latent state representing the participant cognitive state is important. It models the hidden relationship between input variables and feedback estimations. As we assume, modeling human cognitive state influences positively the acoustic and movement productions, which guide then better our DBN-based feedback estimator. We can also observe than this first result outperforms the Decision Tree and MLP classifiers but not yet the SVM classifier (cf. Table 1). The drawback of our first DBN-based strategy is to find the right dimension (or cardinality) for the cognitive state variable in order to model properly the coverbal complexity. Here, the automatic classical DBN training gives a dimension of 5 for the latent states. To go deeper, we investigate 3 clustering methods (i.e. K-means, EM (Expectation-Maximization) and DBSCAN (Density-Based Spatial Clustering of Applications with Noise)) to determine the best cognitive states based on the input training data. For information, Weka Java API [18] has been used to realize the clustering task.

Table 2. Average performances for our first DBN-based feedback estimations (best results in bold format).

\begin{tabular}{llllll}
\hline DBN & & Compact & \multicolumn{3}{l}{ Standard } \\
\hline Granularity & Evaluation Metric & Simple DBN & Latent DBN & Simple DBN & Latent DBN \\
\hline 10s & Precision (\%) & 59.26 & 59.75 & 59.14 & $\mathbf{6 0 . 0 0}$ \\
& F-measure (\%) & 64.69 & 65.43 & 64.57 & $\mathbf{6 5 . 8 0}$ \\
5s & Precision (\%) & 59.57 & 59.44 & 59.20 & 59.14 \\
& F-measure (\%) & 64.32 & 64.38 & 63.95 & 63.83 \\
\hline
\end{tabular}

Table 3 presents our final DBN-based feedback estimations with automatic clustering algorithms to model the cognitive states. The K-mean, EM and DBSCAN clustering give respectively a dimension of 27,8 and 31 for 
CS. The best performances were through the K-means clustering method (with 27 clusters). Particularly for this method, after many empirical tests and exploring cardinalities multiples of the target variable cardinality (i.e. $\{9,18,27,36\})$, we chose the optimal number of 27 clusters, which corresponds to 27 specific Cognitive States (CS). This number has given the best performance among all configurations. About cluster number for K-means algorithm, according to our previous experiences, the number of CS clusters is generally a multiply of the cardinality of the target variable (here the feedback F cardinality). Since F cardinality is 9 , tested numbers for were thus 9, 18, 27 and 36. This way each feedback type will be associated with an equal number of CS comparing to other types. The F-measure of $67.17 \%$ for our DBN with a cognitive state clustered by $27-$ means appears to outperforms all previous results. Designing the right participant cognitive state is then crucial to model temporal input correlations in order to estimate the right feedback to the presenter. Classical classifiers, as Decision Trees, MLP, SVM do not model this hidden structured information.

Table 3. Average performances for our final DBN-based feedback estimations (best results in bold format).

\begin{tabular}{lll}
\hline Granularity 10s (Configuration Standard) & Precision (\%) & F-measure (\%) \\
\hline DBN with CS by K-means (27 clusters) & 62.47 & 67.17 \\
DBN with CS by EM (8 clusters) & 61.36 & 66.91 \\
DBN with CS by DBSCAN (31 clusters) & 57.90 & 64.32 \\
\hline
\end{tabular}

5.1.3 Final Comparison. The final performance comparison is presented in Table 4. For the sake of comparison, we computed also random performances from the empirical distributions of annotations. The random model with weak performances show the complexity of producing the right feedback to participants. According to this table, performances of DBN models are largely higher than random levels. Thanks to CS modeling, our proposed model outperforms the baseline simple DBN (without CS) on both metrics: $62.47 \%$ vs. $59.14 \%$ for precision and $67.17 \%$ vs. $64.57 \%$ for F-measure. It also outperforms the SVM based classifier for feedback estimation $(62.47 \%$ vs. $61.85 \%$ for precision and $67.17 \%$ vs. $65.93 \%$ for the F-measure). This performance gap proves the relevance of our causality network in modeling the studied interaction and confirms the significant contribution of cognitive states in enhancing feedback estimation. Results also show the importance of detecting the adequate cognitive states; otherwise, the DBN modeling will not fulfill its objectives.

Table 4. Average performances for all feedback estimations (best results in bold format).

\begin{tabular}{lll}
\hline Granularity 10s (Configuration Standard) & Precision (\%) & F-measure (\%) \\
\hline DBNs with CS by K-means (27 clusters) & 62.47 & 67.17 \\
Simple DBN without CS & 59.14 & 64.57 \\
SVM & 61.85 & 65.93 \\
Random model & 17.53 & 36.91 \\
\hline
\end{tabular}

5.1.4 Discussion. The presented results in previous subsections are computed from collected data of the 11 participants. At first sight, presenters number may appear moderate, but on the contrary, we think that the data set is sufficiently rich and complex thanks to the multimodality of captured signals and the strictness of the protocol. This should be compared to the exiting data sets in the literature of public speaking evaluations [7]. Moreover, we do think that "the devil is in the details" and that behavior patterns are sufficiently complex for deserving modeling work. This dataset completely fulfills the objective of capturing the micro-structures 
and regularities of speaker behaviors and attitudes. Scaling up statistical models trying to capture interaction is certainly a very challenging task. Before ingesting thousands of hours of interactive and highly multimodal data, we think it is safer and more productive to choose and tune models on carefully designed data. Once the performance of data mining and modeling of such "moderate data set" will reach acceptable performance, it would be relevant to move on to larger sets of observations and cognitive states.

Finally, it is important to signal our awareness of the overfitting problem that may be caused by the moderate number of participants. To cope with this issue, many steps were taken to limit its effect. As a first a step, we applied cross-validation which makes presented metrics as an average result. Surely, cross-validation do not overcome overfitting but can be considered as a first step to make evaluation more appropriate. Secondly, as we used well-known toolboxes in our work, such as Weka for classic classifiers and Bayes Net for DBNs, we sticked to the default regularization parameters of the used algorithms. For instance, to reduce overfitting in Decision Trees, it is recommended to apply pruning on the trained tree. In our case, we set the corresponding parameter ("pruning confidence threshold" to 0.25). For DBNs, Bayes Net toolbox uses the BIC criterion in model selection, which is known to reduce overfitting issues. Moreover, Bayes Net toolbox uses for initialization uniform (uninformative) prior for regularization purposes. In addition, after training we added to CPDs (conditional Probabilities Distributions) a value of 0.001 to remove zero parameters (resulting from sticking to only seen data), which makes the model more efficient and less prone to overfitting.

\subsection{Qualitative results about the experiment design}

One aim behind our study is to (1) survey both presenters and audience about many aspect of the experiment and (2) collect participants' opinions about the quality of our automatic assessment system. Three questionnaires were established to this end: the first one is filled by the speaker just after his/her presentation, the second one is filled by an audience evaluator and the third one is filled by the speaker after getting the automatic evaluation. In this section, we first propose qualitative results about the studied interaction and then qualitative results about the automatic offline scores generated by our rule-based annotation models.

\section{Evaluation from the presenters}

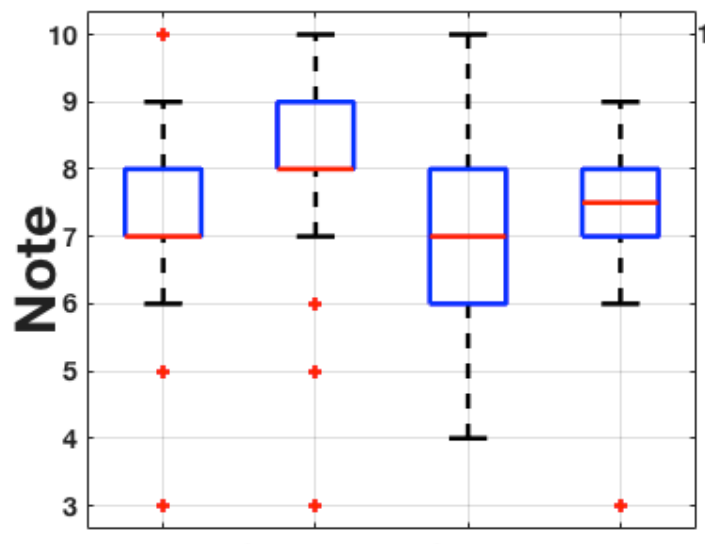

Awareness Concentration Comfort Attention

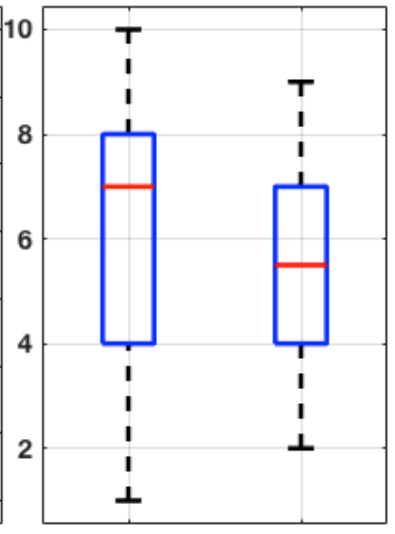

Encumbrance Perturbation

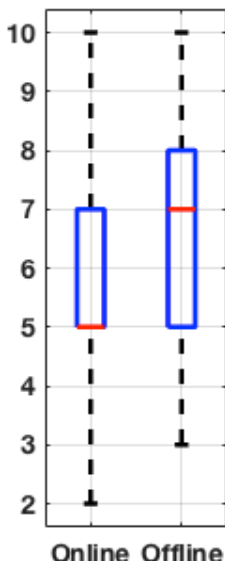

Online Offline

Fig. 7. Evaluations from the presenters. Notation scale was from 1 to 10 (1=total disagreement, $10=$ total agreement). 
Fig. 7. proposes evaluations from the presenters about 8 criteria of the studied interaction. These criteria correspond to the following sub-questions: On a scale of 1 to 10 , do you agree or disagree with the following statements (1 if you totally disagree, 10 if you totally agree):

1. Awareness: "I was aware of my coverbal behavior during my presentation."

2. Concentration: "I was very focused during my presentation."

3. Comfort: "I was comfortable during my presentation."

4. Attention: "I was attentive to audience reactions."

5. Encumbrance: "The experimental devices took up too much space during the presentation."

6. Perturbation: "The experimental devices disrupted my social interaction with the audience."

7. Online Feedback: "To improve my presentations, it will be good to use the experimental devices to get feedbacks about my coverbal behavior during my presentation.”

8. Offline Feedback: "To improve my presentations, it will be good to use the experimental devices to get feedbacks about my coverbal behavior after my presentation.”

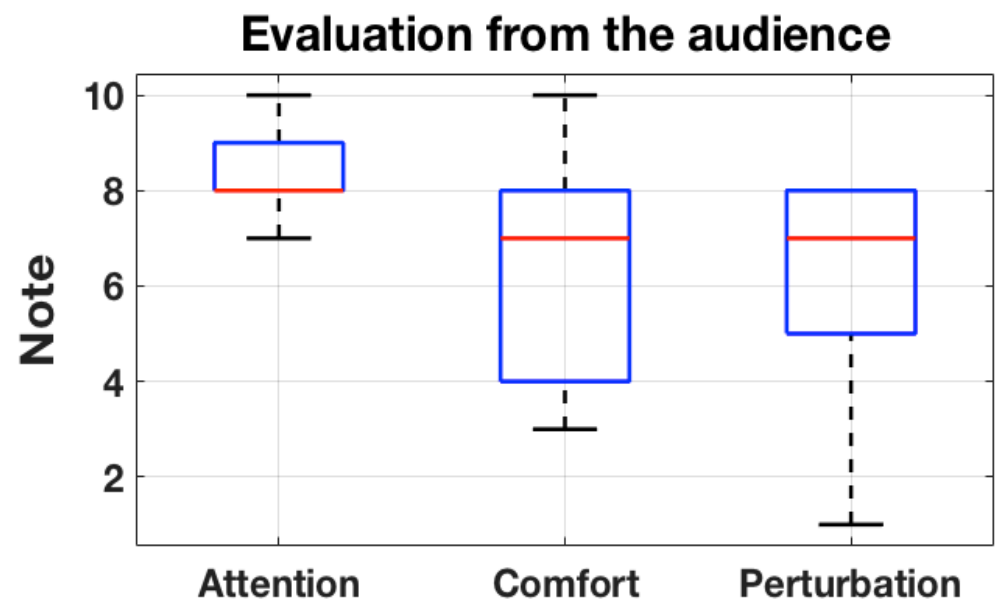

Fig. 8. Evaluations from the audience. Notation scale was from 1 to 10 (1=total disagreement, $10=$ total agreement).

Fig. 8 plots expose relevant values about the audience attention, which respected the experiment instructions. For the other criteria, a mixed feeling is observed. We noticed positive feelings about comfort criterion but also relatively negative ones about perturbation. Indeed, some people complain about the glasses opacity, limiting thus eye contacts. This result joins presenter evaluation about the used glasses.

\subsection{Qualitative results about the proposed offline feedback}

The multimodal signal analysis gave us the opportunity to provide offline feedbacks to the participants about their coverbal behaviors. Fig. 9 presents one of them. For the two sessions (S1 and S2) of the concerned participant, an automatic classification is computed for the six coverbal modalities. First, a learning effect is proved between the two sessions (i.e. for all participants better scores were overall recorded for their second session). In case of testing online feedback system, it will be necessary to exactly quantify learning effect in order to prove that amelioration over time is due to the intelligent system and not simply to a learning effect. For this particular presenter (cf. Fig. 9), the speech analysis presents good performances concerning volume, intonation - even if this dimension could be improvable - and speech rate. In addition, charts show good 
performance on gaze, which indicates that it was oriented to the audience most of the time. Gesture energy was positively evaluated except few excessive segments during the second session. Looking on videos, excessive intervals corresponded to sudden and saccadic gestures performed

Finally, on 10-point scale questions, our participants rated on average 7.45 for the relevance of provided statistics in general, 7.36 for the relevance of those statistics to better understand coverbal behaviors, 6.55 for their relevance to improve presentations, and 6.82 as a motivation level to participate in future experiments with online feedbacks. About the first four criteria, participants have given quite good evaluations, showing in part that the experimenter presentation about coverbal behavior was well perceived. One participant gave the following comment: "My attention was more focused on my body movements during the second session because I was more comfortable".
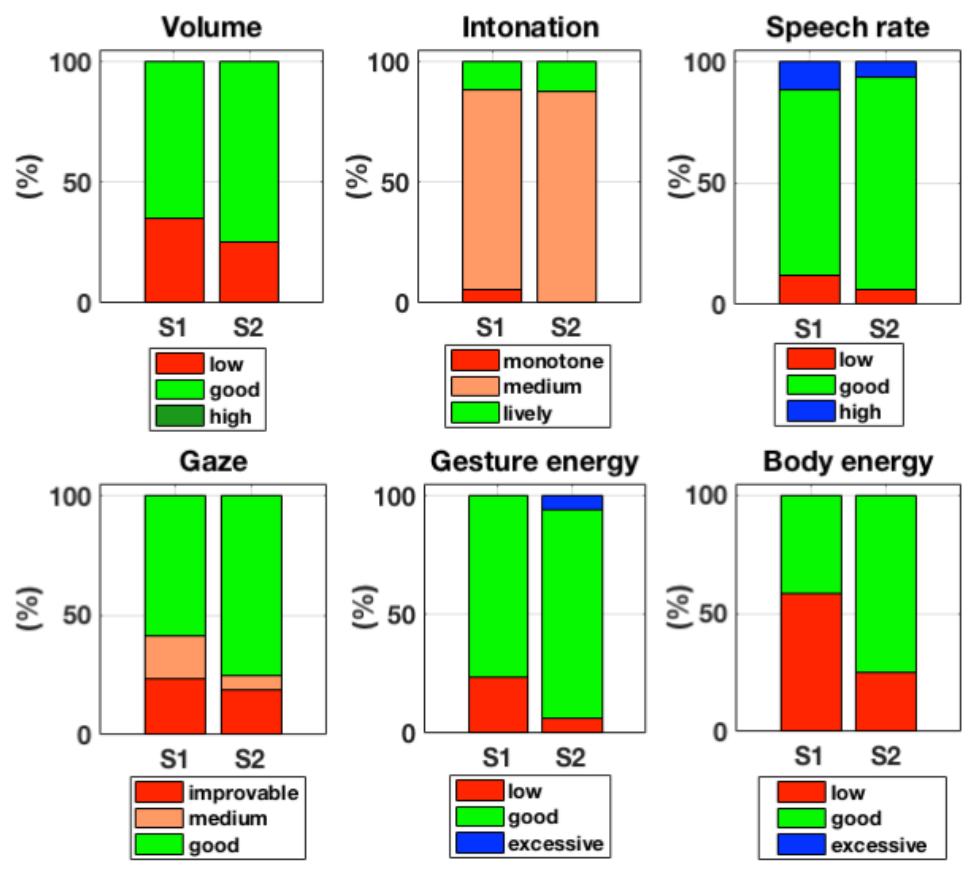

Fig. 9. Offline multimodal scores for one presenter. S1 corresponds to session 1 and $\mathrm{S} 2$ to session 2.

About experimental device perception, statistics shows divided opinions on the perturbation criteria. However, the box plots show relatively high median for the encumbrance criteria, which is due principally to the glasses type. One user explains "I find it extremely heavy". This particular issue would be probably solved in the future thanks to more recent and light devices. Concerning online versus offline feedbacks, the evaluations from the presenters give a preliminarily preference to offline feedbacks. One participant judges online feedbacks as follows: "I imagine it would be too disturbing". Consequently, the challenge for an online assessment system will be to optimize timing and manner of the feedback render. Evaluations from audience evaluators are presented in Fig. 8. They concern 3 criteria that correspond to the following sub-questions:

1. Attention: "I was careful to the coverbal behavior of the presenter."

2. Comfort: "I was comfortable during the presentation even if the presenter wears connected devices."

3. Perturbation: "The experimental setting disrupted social interactions between presenter and audience." 


\subsection{Subjective evaluations by participants}

Fig. 10, Fig. 11 and Fig. 12 show 3 sessions for 3 participants: User A, B and C. The 6 modalities are analyzed automatically by our rule-based models (cf. Section 4.5) and the relative notations are provided respectively by the presenter and an anonymous evaluator from the audience (cf. Section 4.3). The 3 selected speakers represent some special cases. Speaker A and B represent an example where the subjective evaluation meets the automatic assessments. Notice that speaker B exhibits high performance while speaker A exhibits less successful performances. In addition, we added the case of speaker $C$ since it shows an example where the classification was less successful compared to subjective evaluations.

Indeed, user A evaluates the personal behavior with a low mean performance of 5.17/10 (i.e. the average note on all modalities) whereas the audience gives a mean note of $6 / 10$. In contrast, user B gets a good selfevaluation with a mean value of $8.17 / 10$ while the public mean note is 7.83/10. In details, user A (see Fig. 10) is aware about some weakness regarding intonation, gaze, gesture and body energies. These weaknesses are correctly perceived by our automatic classification with for instance $47 \%$ for monotone intonation, $32 \%$ for good gaze, 13\% for correct gesture energy and zero percentage for correct body energy. Otherwise, user B (see Fig. 11) gets strong performances for volume, speech rate, gaze, gesture and body energies according to our automatic classification (cf. percentages on these 5 criteria). Notice that this automatic result is adequate with the personal and the public subjective evaluations.

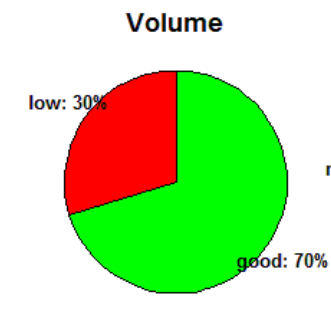

personal note: $7 / 10$ public note: $8 / 10$

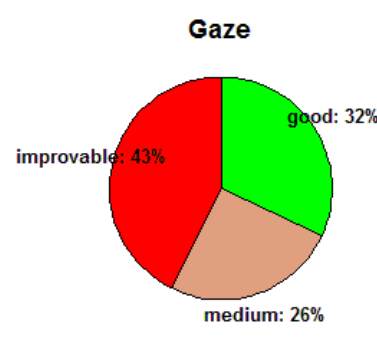

personal note: $6 / 10$ public note: $6 / 10$

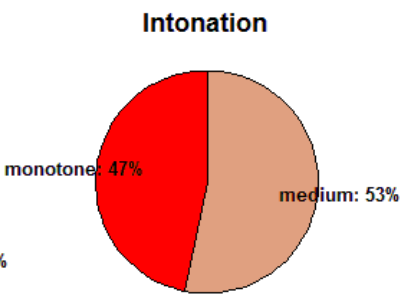

personal note: $5 / 10$ public note: $6 / 10$

Gesture energy

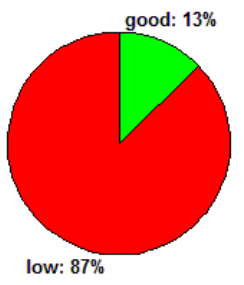

personal note: $4 / 10$ public note: $5 / 10$

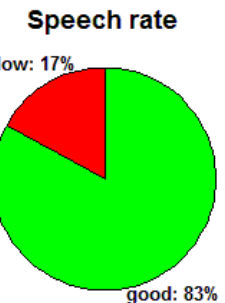

personal note: $6 / 10$ public note: $6 / 10$

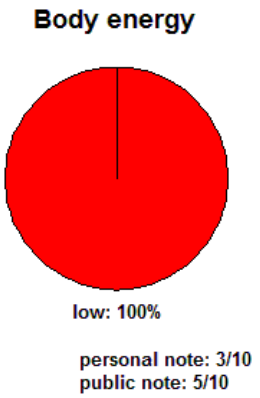

Fig. 10. User A automatic and human evaluations.

In Fig. 12, we expose the results obtained on user $\mathrm{C}$ with less automatic classification success. For instance, the volume is perceived as good for both the presenter and the audience, while the automatic classification assigns a low label to $87 \%$ of the talk. This mismatch is mainly due to the general threshold parameters (cf. Section 4.5.1) that apparently do not match well with this participant in regard to the experimental settings. 

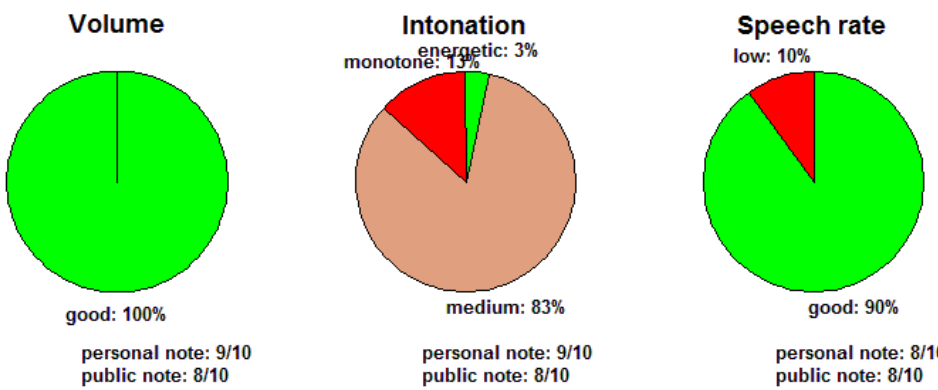

personal note: $8 / 10$ public note: $8 / 10$ public note: $8 / 10$

Gaze

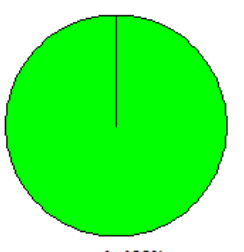

good: $100 \%$

personal note: $8 / 10$ public note: $7 / 10$

\section{Gesture energy}

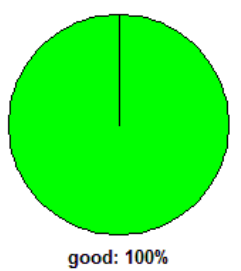

personal note: $8 / 1$ public note: $9 / 10$
Body energy

low: $10 \%$

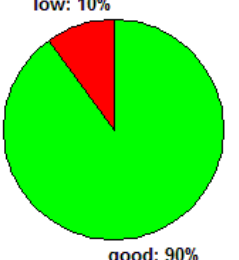

personal note: $7 / 10$ public note: $7 / 10$

Fig. 11. User B automatic and human evaluations.

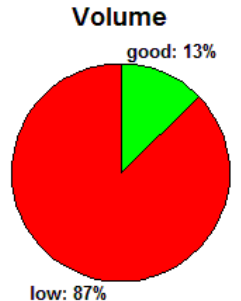

personal note: $7 / 10$ public note: $6 / 10$

Gaze

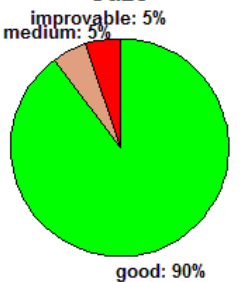

personal note: $9 / 10$ public note: $9 / 10$

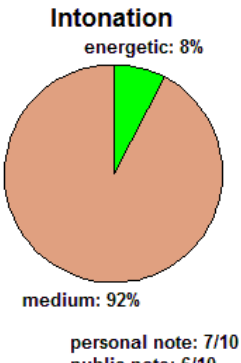
public note: $6 / 10$

Gesture energy

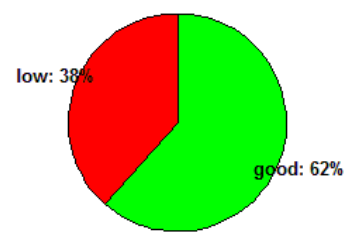

personal note: $6 / 10$ public note: $7 / 10$
Speech rate

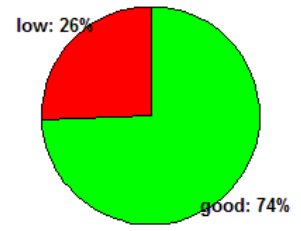

personal note: $6 / 10$ public note: $7 / 10$

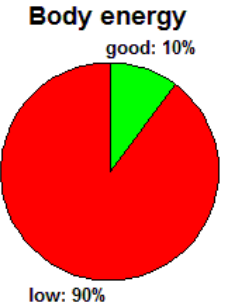

personal note: $3 / 10$ public note: $7 / 10$

Fig. 12. User $\mathrm{C}$ automatic and human evaluations. 
A calibration phase for each participant could be done to tackle this issue. A similar situation appears with the body energy assessment. While both automatic categorization and personal note agree on low performance, the audience evaluator does not seem to be perturbed (the evaluation is equal to $7 / 10$ ). In this case, the mismatch could be simply caused by the subjective view of the evaluator and not the classifier threshold.

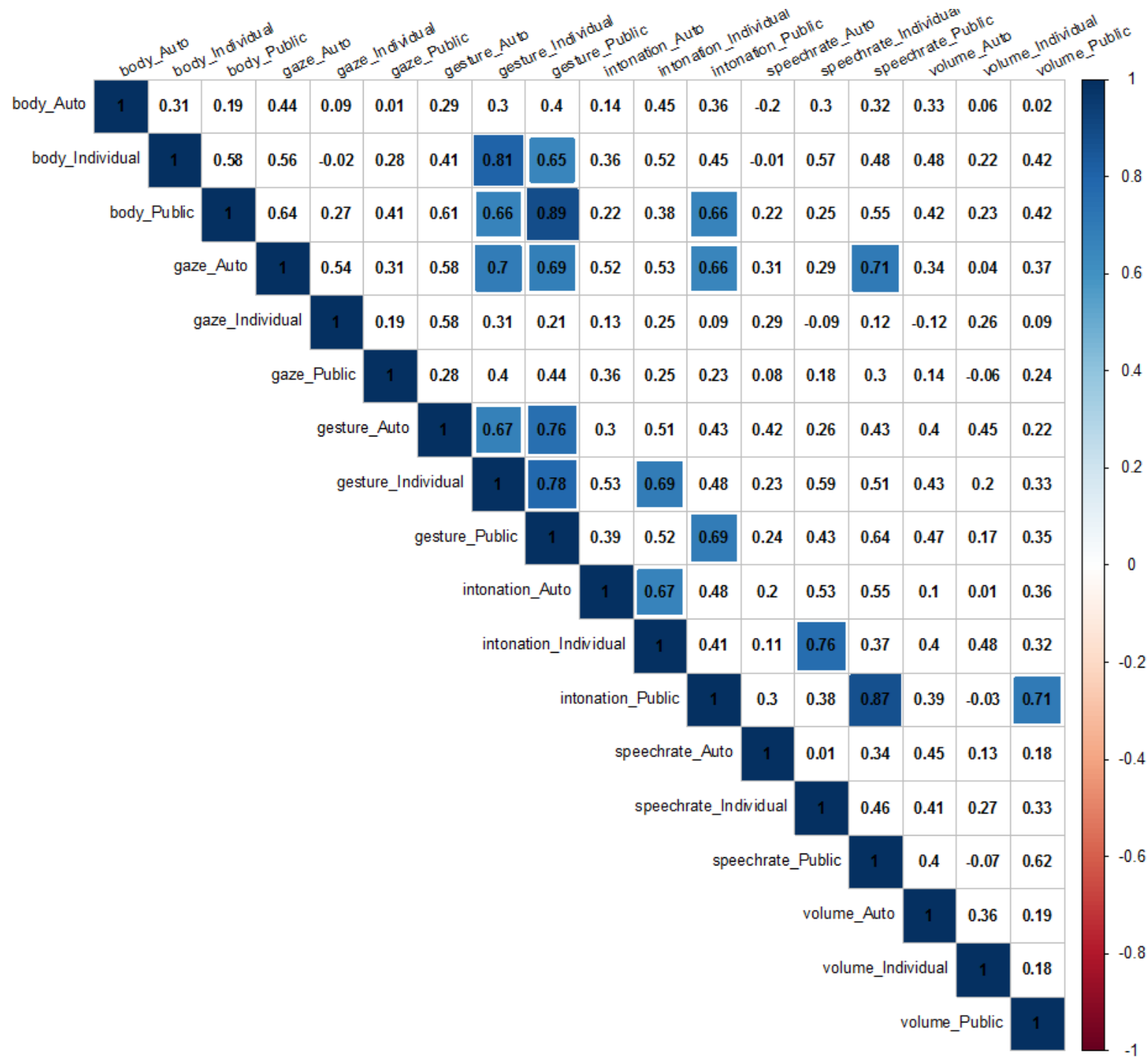

Fig. 13. Significant correlations between automatic, individual and public notations by modalities (colors reveal only the large enough value to be significant).

\subsection{Correlations between automatic, individual and public notations}

To evaluate quantitatively our objective evaluation (versus the subjective one), a correlation study is conducted. Fig. 13 shows the Pearson produce-moment correlation values between the notations provided by each presenter regarding his/her talk (i.e. individual), those provided by the audience when judging a 
particular presenter (i.e. public) and our automatic intelligent system (i.e Auto). All experimental sessions are considered and classified by the 6 modalities. In Fig. 13, only significant values are displayed in color (p-values are inferior or equal to 0.01). This figure illustrates indeed 3 situations. Firstly, some automatic evaluations are well correlated to individual and public perceptions. For instance, the correlation value between automatic and individual gesture evaluations is equal of 0.67 . Likewise, a correlation value of 0.76 is computed between automatic and public gesture notations. These results emphasize the good balance of our automatic gesture classification and human perception. A good match exists also between the automatic intonation notations and the individual evaluations. The correlation value is 0.67 for a p-value equal to $6.81 \mathrm{e}-04$. Nevertheless, some automatic rule-based evaluations seem to not correctly fit with human evaluations, such as body movements, speech rate and volume. These rules may be improved by learning parameters that optimize these correlation relationships.

Secondly, some individual and public evaluations correlate several modalities. The individuals and the audience seem to associate their perception of body and gesture movements. The correlation between gesture and body is of 0.81 for individual evaluations and of 0.89 for public evaluations. Other results are remarkable as the joint perception of the 3 voice modalities for the audience: (intonation_Public, speechrate_Public), (intonation_Public, volume_Public) and (speechrate_Public, volume_Public).

Finally, another interesting correlation exists between individual and public notations regarding gesture perception. The individual and public notations are correlated with a value of 0.78 for a p-value equal to $1.66 \mathrm{e}-$ 05. Clearly, this relation does not apply to all modalities confirming the subjectivity of human evaluation and the differences in perception between presenters and audience about the same modality.

\section{SUMMARY}

In this paper, we studied human behavior analysis in social interactions. Especially, we were interested in modeling nonverbal communication of a public speaker in an oral presentation scenario. The challenge was to develop an automatic assessment system able to assist presenters in becoming brilliant and charismatic speakers. For this purpose and contrary to many works in literature, we designed a DBN-based model to provide relevant feedbacks from only wearable devices and multimodal signal sensing. Using social signal processing techniques, we developed rule-based models that computed performance scores concerning multimodal behavior of the speaker. In our analysis, we proceeded with a multimodal approach that allowed us to model not only voice attributes (volume, intonation, speech rate) but also eye gaze, hand gestures and body movements. Performance scores were communicated to participants in an offline reports (after their presentations) and received positive evaluations about their relevance and their usefulness. Similarly, overall opinions were quite positive about the experimental setting.

In fact, some issues were raised up especially about the heaviness of the smart glasses. This particular issue will be overcome in future research. Other investigations should concern the design and the usability of wearable devices to better collect user behaviors and better inform users about their skills. We know that the precision of the voice, movement and gaze characterization is crucial, but the compactness and the weight of the devices are also relevant in order not to disturb the presenter. Likewise, the way that the interactive and intelligent system should provide feedbacks to users is fundamental. Visual displays are often privileged but audio or tactile information are also more adapted to some public situations. Ergonomic studies will point out the more relevant media inside an interactive loop between users and the intelligent system. User studies should address also a larger participant number and different usage situations (i.e. meetings, seminars, art presentations, etc.). Another interesting topics from the user points of view is to investigate offline and online feedback estimations. Some mistakes are difficult to take into account during the presentation. Consequently, a global investigation could explore how to deliver the right messages but also when it is more appropriate to interrupt the presenter. 
Moreover, one step toward online feedback delivery is ensured thanks to our DBN model, especially with the concern of building a relevant cognitive state variable that links all nonverbal modalities. Indeed, we showed that our proposed causality network (with explicit cognitive state modeling) has led to good performance rates compared to baseline models. Starting from actual models, we will enrich in future our setting with realtime capacities in order to deliver online feedbacks, which enables the speakers to improve in real-time and in-situ their nonverbal communication. A remaining challenge is then to model in our DBN-based system a processing state providing the starting time and the duration of a feedback message to be delivered.

Others learning machine techniques should be investigated in the future such as models using reinforcement learning (for instance POMDP: Partially Observable Markov Decision Process) in order to take into account presenter behaviors to reward or reprimand the learning model. It is also important to let participants be involve during the intelligent system customization with an interactive loop for agreeing or disagreeing the feedback messages in real time. In particular situations, a presenter may want to disable some feedbacks because judged inappropriate due to a current context. This fact guides our research to better integrate also a context management system, understanding more precisely the present situation. Many external parameters influence the current presentation, for instance the level of lighting, the ambient noise, room organization and space, the moment of the day, etc.

Another perspective is to model audience behaviors to inform presenters about public reactions (e.g. paying less attention to the presentation, discussions with colleagues, hands standing up for asking questions, etc.). It would thus be very challenging to design the feed-forward and feedback interactions between the presenter and the audience models, which would allow to go deeper into a mimetic system reflecting real life social interactions.

\section{REFERENCES}

[1] Karl Albrecht. 2006. Social Intelligence: The New Science of Success. John Wiley \& Sons.

[2] Michael Argyle. 1988. Bodily Communication. Routledge, London; New York.

[3] Ray L. Birdwhistell. 1970. Kinesics and Context: Essays on Body Motion Communication. University of Pennsylvania Press, Philadelphia.

[4] Paul Boersma. 2002. Praat, a system for doing phonetics by computer. Glot International 5, 9/10: 341-345.

[5] Mathieu Chollet, Torsten Wörtwein, Louis-Philippe Morency, Ari Shapiro, and Stefan Scherer. 2015. Exploring Feedback Strategies to Improve Public Speaking: An Interactive Virtual Audience Framework. In Proceedings of the 2015 ACM International Joint Conference on Pervasive and Ubiquitous Computing (UbiComp '15), 1143-1154.

[6] Robert G. Cowell, Philip Dawid, Steffen L. Lauritzen, and David J. Spiegelhalter, 2006. Probabilistic networks and expert systems: Exact computational methods for Bayesian networks. Springer Science \& Business Media.

[7] Ionut Damian, Chiew Seng (Sean) Tan, Tobias Baur, Johannes Schöning, Kris Luyten, and Elisabeth André. 2015. Augmenting Social Interactions: Realtime Behavioural Feedback Using Social Signal Processing Techniques. In Proceedings of the 33rd Annual ACM Conference on Human Factors in Computing Systems (CHI '15), 565-574.

[8] Volker Dellwo, Emmanuel Ferragne, and François Pellegrino. 2006. The perception of intended speech rate in English, French, and German by French speakers. In Proc. 3rd International Conference of Speech Prosody, Dresden, Deutschland, $101-104$.

[9] Arthur P. Dempster, Nan M. Laird, and Donald B. Rubin, 1977. Maximum likelihood from incomplete data via the EM algorithm. Journal of the royal statistical society. Series B (methodological), pp.1-38.

[10] Joseph A. DeVito. 2014. The Essential Elements of Public Speaking. Pearson.

[11] Stephen B. Fawcett and L. Keith Miller. 1975. Training public-speaking behavior: an experimental analysis and social validation. Journal of Applied Behavior Analysis 8, 2: 125-135.

[12] Tian Gan, Yongkang Wong, Bappaditya Mandal, Vijay Chandrasekhar, and Mohan S. Kankanhalli. 2015. Multi-sensor SelfQuantification of Presentations. In Proceedings of the 23rd ACM International Conference on Multimedia (MM '15), 601-610.

[13] Daniel Gatica-Perez. 2009. Automatic nonverbal analysis of social interaction in small groups: A review. Image and Vision Computing 27, 12: 1775-1787.

[14] Kevin Gimpel, Daniel Rudoy, Lincoln Laboratory, United States Missile Defense Agency, and United States Air Force. 2008. Statistical Inference in Graphical Models. MIT, Lincoln Laboratory.

[15] Roger Griffiths. 1990. Speech Rate and NNS Comprehension: A Preliminary Study in Time-Benefit Analysis. Language Learning 40, 3: 311-336.

[16] Laura K. Guerrero and Kory Floyd. 2005. Nonverbal Communication in Close Relationships. Routledge, Mahwah, N.J.

[17] Tamas Hacki. 1996. Comparative speaking, shouting and singing voice range profile measurement: physiological and pathological aspects. Logopedics, Phoniatrics, Vocology 21, 3-4: 123-129. 
[18] Mark Hall, Eibe Frank, Geoffrey Holmes, Bernhard Pfahringer, Peter Reutemann, and Ian H. Witten. 2009. The WEKA data mining software: an update. ACM SIGKDD Explorations Newsletter. 11, 1: 10-18.

[19] Rebecca Hincks. 2004. Processing the prosody of oral presentations. In InSTIL/ICALL Symposium 2004.

[20] Rebecca Hincks. 2005. Measures and perceptions of liveliness in student oral presentation speech: A proposal for an automatic feedback mechanism. System 33, 4: 575-591.

[21] Mohammed (Ehsan) Hoque and Rosalind W. Picard. 2014. Rich Nonverbal Sensing Technology for Automated Social Skills Training. Computer 47, 4: 28-35.

[22] Mohammed (Ehsan) Hoque, Matthieu Courgeon, Jean-Claude Martin, Bilge Mutlu, and Rosalind W. Picard. 2013. MACH: My Automated Conversation Coach. In Proceedings of the 2013 ACM International Joint Conference on Pervasive and Ubiquitous Computing (UbiComp '13), 697-706.

[23] Chien-Ming Huang and Bilge Mutlu. 2014. Learning-based Modeling of Multimodal Behaviors for Humanlike Robots. In Proceedings of the 2014 ACM/IEEE International Conference on Human-robot Interaction (HRI '14), 57-64.

[24] Anil K. Jain. 2010. Data clustering: 50 years beyond K-means. Pattern Recognition Letters 31, 8: 651-666.

[25] Nivja H. de Jong and Ton Wempe. 2009. Praat script to detect syllable nuclei and measure speech rate automatically. Behavior Research Methods 41, 2: 385-390.

[26] Michael I. Jordan, ed., 1998. Learning in graphical models (Vol. 89). Springer Science \& Business Media.

[27] Taemie Kim, Agnes Chang, Lindsey Holland, and Alex Sandy Pentland. 2008. Meeting Mediator: Enhancing Group Collaborationusing Sociometric Feedback. In Proceedings of the 2008 ACM Conference on Computer Supported Cooperative Work (CSCW '08), 457-466.

[28] Mark L. Knapp, Judith A. Hall, and Terrence G. Horgan. 2013. Nonverbal Communication in Human Interaction. Wadsworth Publishing, Boston, MA.

[29] Daphne Koller and Nir Friedman. 2009. Probabilistic Graphical Models: Principles and Techniques - Adaptive Computation and Machine Learning. The MIT Press.

[30] Patrick C. Kyllonen. 2012. Measurement of 21st century skills within the common core state standards. In Invitational Research Symposium on Technology Enhanced Assessments. May, 7-8.

[31] Jessica L. Lakin, Valerie E. Jefferis, Clara Michelle Cheng, and Tanya L. Chartrand. 2003. The Chameleon Effect as Social Glue: Evidence for the Evolutionary Significance of Nonconscious Mimicry. Journal of Nonverbal Behavior 27, 3: 145-162.

[32] Jina Lee, Stacy Marsella, David Traum, Jonathan Gratch, and Brent Lance. 2007. The Rickel Gaze Model: A Window on the Mind of a Virtual Human. In Proceedings of the 7th International Conference on Intelligent Virtual Agents (IVA ’07), 296-303.

[33] Vladimir Levenshtein. 1966. Binary Codes Capable of Correcting Deletions, Insertions and Reversals. Soviet Physics Doklady 10, 8 : 707-710

[34] Richard G. Lyons. 2010. Understanding Digital Signal Processing. Prentice Hall, Upper Saddle River, NJ.

[35] Alaeddine Mihoub, Gérard Bailly, Christian Wolf, and Frédéric Elisei. 2015. Learning multimodal behavioral models for face-toface social interaction. Journal on Multimodal User Interfaces: 1-16.

[36] Alaeddine Mihoub, Gérard Bailly, Christian Wolf, and Frédéric Elisei. 2016. Graphical models for social behavior modeling in faceto face interaction. Pattern Recognition Letters 74: 82-89.

[37] Alaeddine Mihoub and Grégoire Lefebvre. 2017. Social Intelligence Modeling using Wearable Devices. In Proceedings of the 22nd International Conference on Intelligent User Interfaces (IUI '17). ACM, New York, NY, USA, 331-341.

[38] Kevin Patrick Murphy. 2001. The Bayes Net Toolbox for MATLAB. Computing Science and Statistics 33: 2001.

[39] Kevin Patrick Murphy. 2002. Dynamic bayesian networks: representation, inference and learning.

[40] Patrick Naïm, Pierre-Henri Wuillemin, Philippe Leray, Olivier Pourret, and Anna Becker. 2007. Réseaux bayésiens. Eyrolles, Paris.

[41] Fumio Nihei, Yukiko I. Nakano, Yuki Hayashi, Hung-Hsuan Hung, and Shogo Okada. 2014. Predicting Influential Statements in Group Discussions Using Speech and Head Motion Information. In Proceedings of the 16th International Conference on Multimodal Interaction (ICMI '14), 136-143.

[42] Hal Pashler. 1994. Dual-task interference in simple tasks: data and theory. Psychological Bulletin 116, 2: 220-244.

[43] Jan Schneider, Dirk Börner, Peter van Rosmalen, and Marcus Specht. 2015. Presentation Trainer, Your Public Speaking Multimodal Coach. In Proceedings of the 2015 ACM on International Conference on Multimodal Interaction (ICMI '15), 539-546.

[44] Richard M. Shiffrin and Gerald T. Gardner. 1972. Visual processing capacity and attentional control. Journal of Experimental Psychology 93, 1: 72-82.

[45] Han Sloetjes and Peter Wittenburg. 2008. Annotation by Category: ELAN and ISO DCR. In Proceedings of the 6th International Conference on Language Resources and Evaluation.

[46] David L. Strayer, Frank A. Drews, and Dennis J. Crouch. 2006. A comparison of the cell phone driver and the drunk driver. Human Factors 48, 2: 381-391.

[47] Md. Iftekhar Tanveer, Emy Lin, and Mohammed (Ehsan) Hoque. 2015. Rhema: A Real-Time In-Situ Intelligent Interface to Help People with Public Speaking. In Proceedings of the 20th International Conference on Intelligent User Interfaces (IUI '15), 286-295.

[48] Toastmasters International. 2011. Gestures: your body speaks. Retrieved from https://www.toastmasters.org/ /media/E202D7AA84E24A758D1BAAE8A77FD496.ashx

[49] Alessandro Vinciarelli, Maja Pantic, Dirk Heylen, Catherine Pelachaud, Isabella Poggi, Francesca D’Errico, and Marc Schroeder. 2012. Bridging the Gap between Social Animal and Unsocial Machine: A Survey of Social Signal Processing. IEEE Transactions on Affective Computing 3, 1: 69-87.

[50] Lilyan Wilder. 1999. 7 Steps to Fearless Speaking. John Wiley \& Sons, New York. 